C2008 IEEE. Personal use of this material is permitted. However, permission to reprint/republish this material for advertising or promotional purposes or for creating new collective works for resale or redistribution to servers or lists, or to reuse any copyrighted component of this work in other works must be obtained from the IEEE. 


\title{
A Survey in Semantic Search Technologies
}

\author{
Hai Dong ${ }^{1}$, Farookh Khadeer Hussain², Elizabeth Chang ${ }^{3}$ \\ ${ }^{1}$ Hai Dong, DEBII, Curtin University of Technology, Perth, Australia, e-mail: hai.dong@cbs.curtin.edu.au \\ ${ }^{2,3}$ Farookh Khadeer Hussain and Elizabeth Chang, DEBII, Curtin University of Technology, Perth, Australia \\ e-mail: (farookh.hussain, elizabeth.chang)@cbs.curtin.edu.au
}

\begin{abstract}
In this paper, we make a survey over the primary literature regarding semantic search technologies. By classifying the literature into six main categories, we review their characteristics respectively. In addition, the issues within the reviewed semantic search methods and engines are analysed and concluded based on four perspectives.
\end{abstract}

Index Terms-semantic search engines, semantic search methodologies, hybrid semantic search, XML search engines, ontology search engines, semantic multimedia search engines.

\section{INTRODUCTION}

Semantic search, as an application of Semantic Web in the field of information retrieval, has shown significant potential in the function of improving the performance of retrieval. Compared with the traditional search engines that focus on the frequency of word appearance, semantic search engines are more likely to try to understand the meanings hidden in retrieved documents and users' queries, by means of adding semantic tags into texts, in order to structuralize and conceptualize the objects within documents [28]. Currently the researches regarding semantic search are in the beginning stage, as the traditional search engines such as Google ${ }^{\circledR}$, Yahoo ${ }^{\circledR}$ and so forth still dominate the markets of search engine.

In this paper, we will make a primary survey over the existing literature regarding semantic search. By classifying the literature into six main categories, we review their characteristics respectively. In addition, the issues within the reviewed semantic search methods and engines are analysed and concluded based on four perspectives.

\section{SeMantic SEARCH}

Current literature with regards to semantic search primarily concentrates on semantic search engines, semantic search methods, hybrid semantic search engines, XML search engines, ontology search engines, and semantic multi-media search engines.

\section{A. Semantic search engines}

Currently, couples of semantic search engines are designed and implemented in order to adapt to different working environments, and the mechanisms that realize these search engines are thus distinct.

Lee and Tsai design an interactive semantic search engine which collects feedback by means of selection in order to better capture users' personal concepts [14]. The search al- gorithm utilized in this model is an "iteratively cyclic mechanism", which includes selecting upper classes and generating lower classes. Selecting upper classes refers to searching for the most suitable web pages by selecting the individuals from the current population and forming a new population; generating lower classes refers to the application of a "genetic operator" on upper classes to generate new lower classes.

Chiang et al. present a semantic search engine based on the smart web query (SWQ) method for web data retrieval [3]. The SWQ architecture contains three main parts: SWQ search engine and its subcomponents: "query parser" and "context ontology determination engine"; context ontologies for domains of application; a semantic search filter which is to improve search precision based on retrieving term properties in context ontologies.

Guha et al. deliver a semantic search engine in TAP - a comprehensive semantic web system [9]. The query language for semantic search in TAP is called the GetData interface, which allows programs to visit properties of a resource in a semantic graph. Each graph is referenced by a URL, and GetData specifies resource name and property name to access to the value of property. Two additional search interfaces are provided by TAP, which are "Search" which searches for any properties with titles containing a given string, and "Reflection" which searches for coming and outgoing tracks for a given node in a semantic graph.

Liu et al. invented an e-service platform integrated with semantic search for e-service metadata [17]. E-service metadata refers to descriptions to e-services and providers, which is to publish and to discover e-services. There are two types of metadata in the system: business level metadata the description of e-service providers, and service level metadata - the description of basic information about e-service. The authors adopt Universal Description, Discovery and Integration which is a web service standard to register and search e-services.

Bhagwat and Polyzotis propose a semantic-based file system search engine - Eureka, which uses an inference model to build the links between files and a FileRank metric to rank the files according to their semantic importance [1]. Eureka has two main parts: (1) crawler which extracts file from file system and generates two kinds of indices: keywords' indices that record the keywords from crawled files, and rank index that records the FileRank metrics of the files; (2) when search terms are entered, the query engine will match the search terms with keywords' indices, and determine the matched file sets and their ranking order by an in- 
formation retrieval-based metrics and FileRank metrics.

Dichev and Dicheva exhibit a view-based semantic search engine in the context of topic-centred learning repository, by means of the extension of the Topic Maps (TM) model which is a lightweight ontology model constructed by topics and relationships between topics [6]. The environment where TM is implemented is TM4L, which is "an environment for building and using ontology-aware learning repositories represented by topics". View in TM is defined as a collection of related topics, occurrences of topics, associations between topics and scopes of topics. The view-based semantic search in TM4L environment includes two phases: transforming a view-based query to a traversal expression and then locating some corresponding resources; using the retrieved resources to locate other relevant resources.

Wang et al. project a semantic search methodology to retrieve information from normal tables, which has three main steps: identifying semantic relationships between table cells; converting tables into data in the form of database; retrieving objective data by query languages [24]. The research objective defined by the authors is how to use a given table and a given domain knowledge to convert a table into a database table with semantics. The authors' approach is to denote the layout by layout syntax grammar and match these denotation with given templates which can be used to analyse the semantics of table cells. Then semantic preserving transformation is used to transform tables to database format.

\section{B. Semantic search methods}

Apart from the implemented search engines above, several semantic search methods in terms of different technologies have been developed.

Ning et al. present a set of ranking algorithms and corresponding search algorithms for the semantic web [20]. The data representation in this research focuses on a weighted directed graph. Based on the graph, the authors develop an extended RDF tuple. For global resource in semantic web, a random surfer can browse ranked objects in terms of the weights of the objects' properties. By means of the basic query and answer search model, the authors develop a general semantic search algorithm based on the graph.

Zhu and $\mathrm{Hu}$ design a semantic search methodology on Distributed Hash Tables (DHT) overlays in the environment of P2P system [27]. Different from traditional DHT structure, the designers add extractor layer and semantic indexing and locating layer between their communications. Two approaches are utilized in the latter layer: LSH-based Semantic Indexing which is to assign same semID for semantically similar files and to cluster semID and nodes with high probability, and LSH-based Semantic Locating which corresponds a query with visiting limited nodes.

Zhang et al. deliver a semantic search methodology in semantic portal. The search model is based on Description Logics (DL)-based information retrieval (IR) [26]. In DL-based IR, the content, the structure, the layout and the thesaurus of a document are stored in the form of DL in knowledge base. A Retrieval Status Value is used to measure the relevance between two documents; a fuzzy-DL is used to assess the uncertainty between the relevance; the concept of fuzzy interpretation is introduced to illustrate a concept and the role of concept in DL.

Liang et al. project a DAML+OIL-based semantic search model [15]. The whole design process can be divided into four phases: (1) designing domain ontology by DAML+OIL; (2) annotating web pages; (3) collecting and storing annotation in web pages; (4) executing searching algorithm. Searching algorithm involves two steps: mapping items to concepts and concept-based search.

Jin et al. address a semantic search schema in P2P network [12]. In a P2P environment, each node contains a number of documents which relate to certain topics. Because of the semantic similarity of documents, there are links between nodes. The search engine is to observe the short-range links between which nodes are semantically similar and the long-range links between which nodes are related in some probability.

\section{Hybrid semantic search engines}

In contrast to the search engines that completely adopt the semantic web technology, some search engines consider the employment of semantic web technology within the keyword-based search engines, in order to improve the precision of traditional text search.

Han and Chen present a hybrid web search methodology - HWS, combining traditional text search and semantic search, to improve the performance of traditional search engines [10]. Three algorithms are adopted in the search engine, namely BAS which is used to mine associations from existing user profile ontologies, $\mathrm{BCH}$ which is to construct class hierarchies by means of hierarchy cluster method, and $\mathrm{MCH}$ which merges classes into class hierarchies. Ranking algorithm utilized in HWS concerns all entities and relations, and contextual similarities between two entities.

Rocha et al. build a hybrid search engine combining traditional text search methodology and a spread activation algorithm over semantic web [22]. Three different measures are counted for weighting relationships between nodes in a semantic graph: (1) cluster measure which is to assess the similarities between nodes; (2) specificity measure which is to assess the differentiation or specificity between nodes; and (3) combined measure which integrate the former two measures.

Kandogan et al. develop a semantic search engine Avatar, which combines the traditional text search engine with use of ontology annotations [13]. Avatar has two main functions: (1) extraction and representation - by means of UIMA framework, which is a workflow consisting of a chain of annotators extracted from documents and stored in the annotation store; (2) interpretation - a process of automatically transforming a keyword search to several precise searches. Avatar consists of two main parts: semantic optimizer and user interaction engine. When a query is entered into the former, it will output a list of ranked interpretations for the query; then the top-ranked interpretations are passed to the latter, which will display the interpretations and the retrieved documents from the interpretations. 
Cesarano et al. develop an agent-based semantic search engine [2]. The whole search procedure contains: (1) users submit a query to user interface combined with a depth parameter which means the search scope, a language selection of queried web pages plus a context option for search area; (2) then these parameters are passed into a traditional search engine to execute search; (3) results involving hyperlink are returned by traditional search engine; (4) the web spider (WS) downloads all pages by hyperlink and then visits the pages which traditional search engine cannot reach; (5) the WS stops when predefined depth parameter is reached; (5) the document pre-processor extracts useful information for each downloaded page; (6) the miner agent ranks these pages by a semantic knowledge base according to the similarities between the pages' information and predefined search context.

\section{XML search engines}

Many search engines are adopted in the purpose of querying objects in XML documents.

Luk et al. categorize four types of XML search. The first is full-text search, which sees XML documents as a collection of structured texts, and then executes a series of algebra query language to retrieve (e.g. sgrep) [18]. The second is to filter information to discard. The third is XML-assisted search, which has several applications: one is to encode loose information to structured information for semantic search; another is to use XML to translate queries and search results among distributed search engines for search collaboration, or to use XML to facilitate information transformation among different search engines. Xyleme project is one of the instances in which queries can be made against the views of XML document hierarchy. The fourth is multistage search namely dividing the search process into multiple phases which have several types.

Chu-Carroll et al. propose to utilize XML fragments to improve the semantic search precision [4]. Three XML fragments are applied to semantic query, to realize the function of conceptualization, restriction and relation respectively. Conceptualization fragment is to generate a lexical string to represent a concept; restriction fragment is to set conditions (string) for XML tags; relation fragment is to show the relationship between two concepts.

Cohen et al. design a framework to describe the relationships between tagged nodes in XML documents [5]. In the framework, an interconnection semantics is derived for querying relationships between XML data based on keywords. The designers assign each node in XML document with an ID reference, thus the distribution of XML node forms a graph structure compared with the tree structure in past researches, which can contain more complex relationships in XML documents. The authors then utilize several semantics to infer the correct relationship between nodes. Three approaches are utilized to realize the objective: (1) a framework for users and creators of documents to define interconnection semantics in regards to the semantic relationship between two nodes; (2) various interconnection semantics are derived from the framework to improve the recall; (3) all queried answers are evaluated by an enumera- tion algorithm, and an algorithm is given to test the validity of the queried semantic relationships to improve the precision.

Syeda-Mahmood et al. develop a semantic search mechanism for XML repositories [23]. A two-step ranking algorithm is given for ranking a XML schema, which counts on both the cardinality and the similarity of the matched query attributes and the matched repository schema attributes. The authors compute similarity based on both names and types of attributes. For the schema indexing, the authors design a type hash methodology, to hash the attributes together with the same feature list, which forms a hash table that maintains an indexing schema of these attributes. When a query attribute is given, the matched attributes from the same feature list are obtained and indexed by the hash table.

\section{E. Ontology search engines}

Some search engines are designed for querying ontological files.

Maedche et al. design an integrated approach for ontology searching, reusing and update [19]. In its architecture, an ontology registry is designed to store the metadata about ontologies and ontology server stores the ontologies. The ontologies in distributed ontology servers can be created, replicated and evolved. Ontology metadata in ontology registry can be queried and registered when a new ontology is created. Ontology search in ontology registry is executed under two conditions - query-by-example is to restrict search fields and search terms, and query-by-term is to restrict the hyponyms of terms for search.

Swoogle is a crawler-based semantic search engine [8]. Three main functions are provided by Swoogle, which are finding appropriate ontologies for specific terms involved; finding instance data - semantic web documents defined by specific classes and properties; characterizing the semantic web - by gaining interrelationship among metadata in semantic web, Swoogle is able to answer the questions about semantic web structure.

Petel et al. develop a semantic web portal - OntoKhoj, in the purpose of ontology search, ranking and classification [21]. The ontology classification model utilized is derived from DMOZ, which is a great number of manually created ontological concept hierarchies. When a new ontology is retrieved, the ontology will be matched with the model to decide which discipline it belongs to. An OntoRank algorithm is designed based on the PageRank algorithm, to rank the retrieved ontologies for a given topic. The difference between PageRank and OntoRank is that the latter does not only take account of the number of references between ontologies, but also includes the weight of the references between ontologies.

\section{F. Semantic multi-media search engines}

The utilization of semantic search also spreads into the field of multi-media. In the following literature, several distinctive semantic search engines in the purpose of retrieving audios [16], flash movies [7] and images [11] [25] will be described respectively.

Linckels et al. propose an ontology-based lecture audio 
search engine in the field of e-librarian service [16]. The e-librarian service platform consists of a domain ontology, a mechanism to identify learning objects (recorded lectures) and a semantic search engine which returns answers of natural language for given questions. The authors deploy OWL-DL to represent the domain knowledge. In DL, the representation form of knowledge consists of concepts, roles (relations) and other constructors such as inheritance, conjunction and extensional restriction. A mechanism is developed to identify learning objects in lecture. First of all, a speech recognition software converts all lecture audios to transcripts; the transcripts are transformed to unified format with part-of-speech tags; the learning objects are identified from the unified transcript. The learning objects can be queried by the semantic search engine.

Ding et al. propose a semantic flash search engine, by means of creating an expressive semantics for flash movies [7]. The ranking algorithm is based on an eigenvector model which consists of two concepts: common expression and well defined movie. Thus, the authors deduct that the above concepts can mutually and positively enhance each other. In addition, search engine can query movies by keywords from the former; and rank the movies based on the later. An expression matrix, composed of expression vectors and movies, is designed to analyse the relationship between them and thus used for semantic rank.

Hoi and Lyu propose a semantic image search schema, by means of learning concepts from image descriptions [11]. Wang et al. propose an image search engine - IGroup, by means of semantic clustering technique [25]. With IGroup, users can refine query results by a navigation panel which contains a list of clusters, and each cluster is represented with a thumbnail and a cluster name.

\section{Some Common IsSues}

We have provided a preliminary survey of the existing and dynamic area in semantic search engines and methods. Although we have not claimed this survey is comprehensive, some common issues in the current semantic search engines and methods are concluded as follows:

\section{A. Differentiation between designers and users' percep- tions}

Concept hierarchy structures in the semantic search engines are not able to completely satisfy users' requirements due to the differences between each person's subjective perceptions regarding the objective world.

For instance, in the RSS model, the transition probabilities distribution is a subjective process, subjecting to the designers' own perception to the relevant importance of relationship between subjects and objects [20]. The designers' perception and users' perception to such relationships are not able to be unified due to the difference of humans' understanding to any items in the real world. The global ranking values on resource are configured by the designers, which has the probability of unacceptableness by users, since the definitions of importance between normal users and the designers could have a huge gap. Without the function of customization, the global ranking values cannot be convincingly built.

In Lee's model, since the utilized matching algorithm cannot completely reflect the semantic similarity of web page content, users' requests could not be completely satisfied [14]. The frequencies of words are not directly relevant to users' concepts in mind, thus the ranking of web pages based on the word lists in the profiles could not completely match users' requests.

In the SWQ search engine, the number of context ontologies could be limited and cannot satisfy users' requests if the context ontologies are designed by the designers [3].

\section{B. Static knowledge structure}

Knowledge structures in the semantic search engines cannot be frequently updated to suit the change of users' requirements and the change of external environment.

For instance, in the HWS search engine, the class hierarchies cannot be modified by either users or designers, which cannot adapt to the dynamic knowledge structure in the real environment and satisfy users' structure change requests [10].

In the SWQ search engine, the context ontologies cannot be customized and updated, which could not satisfy users' changing requests and adapt to the dynamic environment [3].

In the TAP search engine, its knowledge structure in knowledge database cannot be customized, which could lead to the out-of-date knowledge structure that cannot catch up the change of environment and users' requirements [9].

In Liu's e-service platform, the concept hierarchies could not be updated to adapt the change of environment [17].

In the semantic protal model, the retrieval status values that are used to measure the relevance between two documents cannot be customized by users, which cannot satisfy users' needs [26].

In Cesarano's search model, the semantic knowledge base could not be updated to satisfy users and environment's dynamic requirements [2]. The relevance between concepts cannot be customized by users, which cannot reveal users' own understanding towards queried concept.

\section{Low precision and high recall}

Some hybrid semantic search engines cannot show their significant performance in improving precision and lowering recall.

For instance, the HWS hybrid search engine has the issue of query flooding for each keyword in one query and thus could improve the cost of search time and resource, and increase the browsing time of users. The search precision value in the experiment is around $77 \%$, which is not outstanding and obviously preponderant compared with other search methodologies [10].

In Chu-Carroll's XML search engine, the experiment about disambiguation shows that there is no significant improvement being performed compared with the original search method [4].

In Ding's semantic flash search engine, the resource of the search engine is based on the top-50 returned results from Google that is not a semantic search engine, which could cause low recall and low precision [7]. 
In Hoi's image search engine, the experiment shows that the precision of preliminary search results is from $14.5 \%$ to $5.7 \%$ in the range of top- 20 s to top- $100 \mathrm{~s}$, which is a poor result for search engine evaluation; even after several improvements by feedback technique, the highest precision is less then $60 \%$, which is not considered as a strong proof for the feasibility of the search engine [11].

\section{Lack of experimental tests}

Since many semantic search methodologies are only in the phase of conceptual model development, many of them have not been tested via the reasonable number of experiments.

For example, in Lee's search model, the effectiveness of users' feedbacks is tested by only three queries in a case study, which is limited in amount and cannot solidly prove the validity of the conceptual model [14].

In Rocha's hybrid search engine, the instances used in test are limited, which cannot reveal the real feasibility of the search engine [22].

In Cesarano's search model, the number of queries in empirical test is limited, which cannot reveal the feasibility of the prototype in practice [2]. Moreover, there is no test to prove its feasibility in the semantic search within English-based environment.

In Wang's semantic search method, the number of cases in the experiment is not enough (10-13/table type), which needs more experiments to prove its feasibility [24]. Furthermore, there is no criterion given in the experiment, which cannot prove its reasonability.

In the OntoKhoj ontology search model, due to the limited number of the experimental ontologies, the model has not been completely validated [21].

In the Avatar search engine, the Eureka search engine, Cohen's XML search engine and Dichev's view-based search model, there is no empirical test being made to prove its feasibility [1] [5] [6] [13].

\section{CONCLUSION}

In this paper, we make a brief survey over the existing literature regarding semantic search technologies. By classifying the literature into six main categories - semantic search engines, semantic search methods, hybrid semantic search engines, semantic XML search engines, semantic ontology search engines and semantic multimedia search engines, we review their characteristics respectively. In addition, the issues within the reviewed semantic search methods and engines are concluded based on four perspectives - differentiations between designers and users' perceptions, static knowledge structure, low precision and high recall and lack of experimental tests.

In the future, our work will focus on the deeper and broader research in the field of semantic search, with the purpose of concluding the current situation of the field and promote the further development of semantic search technologies.

\section{REFERENCES}

[1] D. Bhagwat and N. Polyzotis, "Searching a file system using inferred semantic links," in Proceedings of HYPERTEXT'05 Salzburg, 2005, pp. 85-87.

[2] C. Cesarano, A. d'Acierno, and A. Picariello, "An intelligent search agent system for semantic information retrieval on the internet," in Proceedings of WIDM '03 New Orleans, 2003, pp. 111-117.

[3] R. H. L. Chiang, C. E. H. Chua, and V. C. Storey, "A smart web query method for semantic retrieval of web data," Data \& Knowledge Engineering, vol. 38, 2001, pp. 63-84.

[4] J. Chu-Carroll, J. Prager, K. Czuba, D. Ferrucci, and P. Duboue, "Semantic search via XML fragments: a high-precision approach to IR," in Proceedings of SIGIR '06 Seattle, 2006, pp. 445-452.

[5] S. Cohen, Y. Kanza, B. Kimelfeld, and Y. Sagiv, "Interconnection semantics for keyword search in XML," in Proceedings of CIKM '05 Bremen, 2005, pp. 389-396.

[6] C. Dichev and D. Dicheva, "View-Based Semantic Search and Browsing," in Proceedings of WI '06, 2006.

[7] D. Ding, J. Yang, Q. Li, L. Wang, and W. Liu, "Towards a flash search engine based on expressive semantics," in Proceedings of WWW Alt. '04 New York, 2004, pp. 472-473.

[8] L. Ding, T. Finin, A. Joshi, R. Pan, R. S. Cost, Y. Peng, P. Reddivari, V. Doshi, and J. Sachs, "Swoogle: a search and metadata engine for the semantic web," in Proceedings of CIKM'04 Washington, 2004, pp. 652-659.

[9] R. Guha and R. McCool, "TAP: a Semantic Web platform," Computer Networks, vol. 42, 2003, pp. 557-577.

[10] L. Han and G. Chen, "The HWS hybrid web search," Information and Software Technology, vol. 48, 2006, pp. 687-695.

[11] C.-H. Hoi and M. R. Lyu, "Web image learning for searching semantic concepts in image databases," in Proceedings of WWW Alt. '04 New York, 2004, pp. 406-407.

[12] H. Jin, X. Ning, and H. Chen, "Efficient search for peer-to-peer information retrieval using semantic small world," in $W W W^{\prime} 06$ Edinburgh, 2006, pp. 1003-1004.

[13] E. Kandogan, R. Krishnamurthy, S. Raghavan, S. Vaithyanathan, and H. Zhu, "Avatar semantic search: a database approach to information retrieval," in Proceedings of SIGMOD '06 Chicago, 2006, pp. 790-792.

[14] W.-P. Lee and T.-C. Tsai, "An interactive agent-based system for concept-based web search," Expert Systems with Applications, vol. 24, 2003, pp. 365-373.

[15] B. Liang, J. Tang, J. Li, and K. Wang, "Using DAML+OIL to enhance search semantic," in Proceedings of WI '04 2004.

[16] S. Linckels, S. Repp, N. Karam, and C. Meinel, "The virtual tele-tASK professor: semantic search in recorded lectures," in Proceedings of SIGCSE '07, Covington, 2007, pp. 50-54.

[17] D.-R. Liu, M. Shen, and C.-T. Liao, "Designing a composite e-service platform with recommendation function," Computer Standards \& Interfaces, vol. 25, 2003, pp. 103-117.

[18] R. W. P. Luk, H. V. Leong, T. S. Dillon, and A. T. S. Chan, "A survey in indexing and searching XML documents," Journal of the American Society for Information Science and Technology, vol. 53, 2002, pp. $415-437$

[19] A. Maedche, B. Motik, L. Stojanovic, R. Studer, and R. Volz, "An infrastructure for searching, reusing and evolving distributed ontologies," in Proceedings of $W W W^{\prime} 03$ Budapest, 2003, pp. 439-448.

[20] X. Ning, H. Jin, and $\mathrm{H}$. Wu, "RSS: A framework enabling ranked search on the semantic web," Information Processing \& Management, 2007 , in press.

[21] C. Patel, K. Supekar, Y. Lee, and E. K. Park, "OntoKhoj: a semantic web portal for ontology searching, ranking and classification," in Proceedings of WIDM '03 New Orleans, 2003, pp. 58-61.

[22] C. Rocha, D. Schwabe, and M. P. Aragao, "A hybrid approach for searching in the semantic web," in Proceedings of $W W W{ }^{\prime} 04$ New York, 2004, pp. 374-383.

[23] T. Syeda-Mahmood, G. Shah, L. Yan, and W. Urban, "Semantic search of schema repositories," in Proceedings of $W W W$ '05 Chiba, 2005, pp. 1126-1127.

[24] H. L. Wang, S. H. Wu, I. C. Wang, C. L. Sung, W. L. Hsu, and W. K. Shih, "Semantic search on Internet tabular information extraction for answering queries," in Proceedings of CIKM '00 McLean, 2000, pp. 243-249.

[25] S. Wang, F. Jing, J. He, Q. Du, and L. Zhang, "IGroup: presenting web image search results in semantic clusters," in Proceedings of CHI '07, San Jose, 2007, pp. 587-596. 
[26] L. Zhang, Y. Yu, J. Zhou, C. Lin, and Y. Yang, "An enhanced model for searching in semantic portals," in Proceedings of $W W W^{\prime} 05$, Chiba, 2005, pp. 453-462.
[27] Y. Zhu and Y. Hu, "Efficient semantic search on DHT overlays," Journal of Parallel and Distributed Computing, vol. 67, 2007, pp. 604-616.

[28] R. Guha, R. McCool, and E. Miller, "Semantic search," in Proceedings of $W W W^{\prime} 03$, Budapest, 2003, pp. 700-709. 